\title{
Fat in the Abdomen Area as a Propagation Medium in WBAN Applications
}

\author{
Mariella Särestöniemi ${ }^{1}$, Carlos Pomalaza Raez ${ }^{2}$, Chaïmaâ Kissi ${ }^{3}$, Timo Kumpuniemi ${ }^{1}$, \\ Marko Sonkki ${ }^{1}$, Matti Hämäläinen ${ }^{1}$, Jari Iinatti ${ }^{1}$ \\ ${ }^{1}$ Centre for Wireless Communications, University of Oulu \\ ${ }^{2}$ Department of Electrical and Computer Engineering, Purdue University \\ ${ }^{3}$ Electronics and Telecommunication Systems Research Group, National School of Applied \\ Sciences (ENSA), Ibn Tofail University
}

\begin{abstract}
This paper presents a study on the fat in the abdomen area as a propagation medium in wearable and implant communications systems. Propagation via subcutaneous and visceral fat is considered separately. Simulations and measurements are done for both female and male bodies with the on-body antennas designed for in-body communications. Propagation paths are calculated and compared with the simulated and measured impulse responses. Furthermore, we analyze simulated 2D power flow figures, which illustrate the propagation inside the different tissues. It is shown that the signal propagates through the fat layer with minor losses compared to the other tissues of the studied cases. The signal propagates through the fat tissue from the abdomen area to the backside of person with $60 \mathrm{~dB}$ power loss. Additionally, the calculated fat layer propagation paths match well with the peaks of the simulated and measured impulse responses. The information about the fat as propagation medium is useful when designing the wireless and wired medical and health monitoring devices.
\end{abstract}

Keywords: anatomical voxel model, capsule endoscopy, directive antenna, finite integration technique, gastrointestinal monitoring, implant communications, power distribution, radio channel, wireless body area networks

\section{Introduction}

Recently, wearable and implant communications have been intensively studied topics in the wireless body area networks (WBAN) [1]-[4]. Smooth design of the communications systems requires comprehensive knowledge of the radio channel characteristics. Hence, in-body channel modeling and propagation within the tissues have been under the scope in e.g. in [5]-[17].

It has been recognized that the signal propagates differently in different tissues due to the variations in dielectric properties [18]. Fat tissue appears to be the easiest tissue for the propagation in terms of propagation velocity and losses [5, 6, 10, 19-21]. Fat as a propagation channel has been a topic for few recent papers [19-21], which have verified by simulation and measurement based studies that the fat tissue is promising propagation medium is the ultra wideband (UWB) based medical applications.

Ref. [10] presents a study on the in-body power distribution for abdominal monitoring and implant communication systems. The study is conducted by examining power flow distribution on the anatomical voxel model at different cross section lay- 
ers in the abdomen area. The presented power results verify that the signal travels through the fat layer for wider areas with minor power loss than in the other tissues. This paper is a continuation for the power distribution studies presented in [10], focusing now on the propagation in the fat layer. The aim of this paper is to present a study on the fat as a propagation medium in the abdomen area using finite integration technique (FIT) based simulations using an anatomical voxel models. Furthermore, measurement data and propagation path calculations are used in the analysis to validate the results. Both subcutaneous fat and visceral fat are considered separately.

This paper is organized as follows: Section II presents the study cases describing the simulations, measurements, antennas, and antenna locations. Furthermore, propagation path calculations are described as well. Section III presents the propagation through the fat layer with 2D power flow diagrams. Section IV presents propagation through subcutaneous fat layer with measurement based studies and propagation path calculations. Conclusions are given in Section V.

\section{Study Case}

\subsection{Simulations}

Simulations are conducted with electromagnetic simulation software CST MicroWave Studio [22] by using finite integration technique (FIT). CST provides several voxel models, among which we selected anatomical voxel models Laura and Gustav, presented in Fig. 1a-b. Laura correspond to lean female body with resolution of $1.875 \mathrm{~mm} \times 1.875 \mathrm{~mm} \times 1.25 \mathrm{~mm}$. Gustav is a normal-weighted male with the resolution of $2.08 \mathrm{~mm} \times 2.08 \mathrm{~mm} \times 2 \mathrm{~mm}$. Cross section of the voxel models abdomen area on the navel line is shown in Fig. 1c. Subcutaneous and visceral fat, muscles, small intestine (SI), and colon are marked in the Fig. 1c.
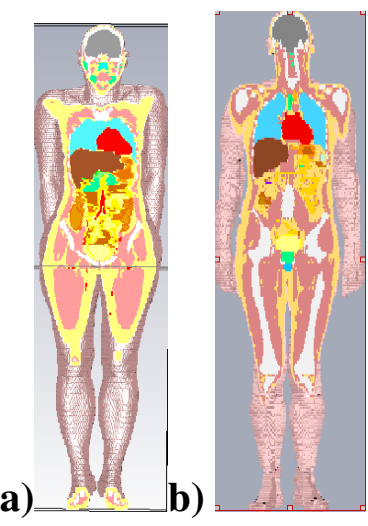

c)

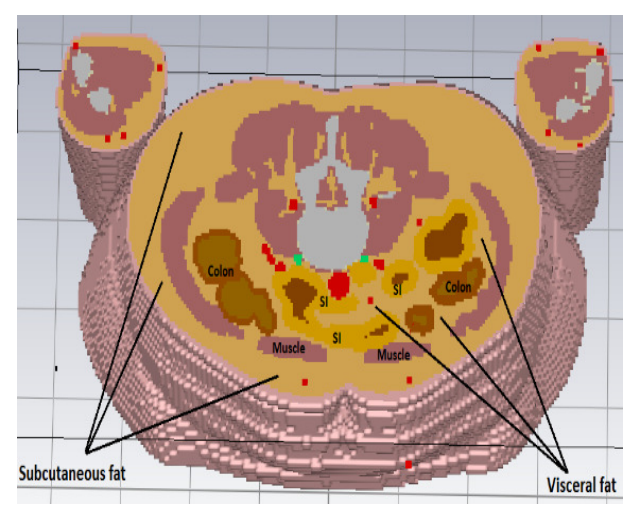

Fig. 1 a) Anatomical voxel model Laura, b) Gustav, c) cross cut of the abdomen area presenting the subcutaneous and visceral fat. 


\subsection{Measurements}

The measurements were conducted in an anechoic chamber in University of Oulu by using Agilent $8720 \mathrm{ES}$ vector network analyzer (VNA). The measured frequency band was the full UWB band $3.1-10.6 \mathrm{GHz}$, according to IEEE802.15.6 standard [23]. We also used a large bandwidth to achieve good time resolution for the measurements. Transmitted power was selected to be $+5 \mathrm{dBm}$, which does not cause harm for human tissues.

The antenna prototypes were connected to the VNA by using $8 \mathrm{~m}$ long phase stable Huber+Suhner SUCOFLEX 104PEA measurement cables. A proper calibration was performed before the measurements to neglect cable effect. The VNA was set to sweep 100 times for each scenario, collecting 1601 frequency points per sweep for each measurement setup. The measurements were conducted in the frequency domain to obtain radio channel frequency responses (S21 parameters), which were later transformed into the time domain in Matlab using inverse fast Fourier transform (IFFT). After IFFT, radio channel impulse response (IR) is obtained.

In this paper, we use the measurement data of two human volunteers, one male (M3) and one female (F1), with different body sizes and body balances. The details of the volunteers are presented in [7], but here we repeat the information in Table I, which is relevant for the propagation studies through the fat layer.

Table I. Information about the volunteers.

\begin{tabular}{|l|c|c|c|c|}
\hline Volunteer & $\begin{array}{c}\text { Waist } \\
{[\mathrm{cm}]}\end{array}$ & $\begin{array}{c}\text { Visceral } \\
\text { fat [\%] }\end{array}$ & $\begin{array}{c}\text { Overall } \\
\text { fat [\%] }\end{array}$ & $\begin{array}{c}\text { Muscle } \\
{[\%]}\end{array}$ \\
\hline $\begin{array}{l}\text { Female } \\
\text { F1 }\end{array}$ & 76 & 5 & 29 & 34 \\
\hline Male M3 & 103 & 14 & 32 & 31 \\
\hline
\end{tabular}

\subsection{Antenna and antenna location}

In this study case, we use a cavity-backed low-band UWB on-body antenna designed for in-body communications. The antenna's simulation model and the prototype are presented in Fig 2a-b, respectively [24]. On the studied simulation and measurement scenarios, transmitter (Tx) antenna is on the navel, and the receiver $(\mathrm{Rx})$ antenna is on the flank. This scenario is conceivable for abdominal monitoring scenarios. 
a)

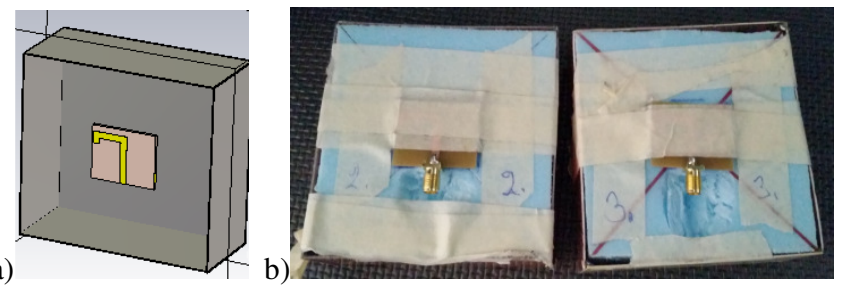

c)

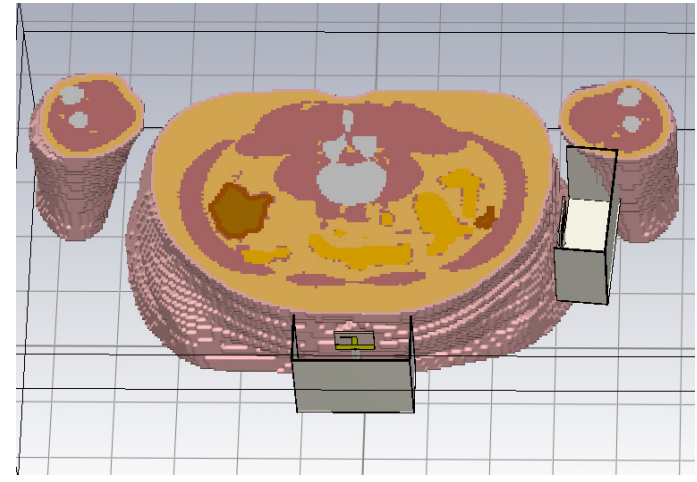

Fig 2. Cavity-backed low-band UWB antenna designed for in-body communications, b) prototypes used in the measurements, c) location of the antennas

\subsection{Propagation path calculations}

In [5], we presented the idea for calculating the propagation paths when signal is passing through different tissues. In this paper, we just focus on the propagation paths through the fat layer, either subcutaneous or visceral fat - or as combination. As discussed in [5], the propagation time $t_{\mathrm{d}}$ is calculated taking into account the frequency $f$, the distance $d$ that the signal travels through tissue, and the wavelength in the tissue $\lambda$ as

$$
t_{d}=\frac{d}{v}=\frac{d}{f \lambda}
$$

Wavelengths on the different tissues are presented in Table II. As one can note, wavelength, and hence the propagation speed $v$ in the tissue may vary significantly between different tissues. For instance, wavelength in the fat tissue is remarkable different from other tissues. Hence, the propagation speed is much higher in fat than for instance in muscle layer. The Table II includes also the estimated thicknesses of the tissue layers on the navel area of the voxel model. 
TABLE II: Thickness of the layers on the navel area and wavelengths in the tissues.

\begin{tabular}{|l|l|l|}
\hline Tissue & $\begin{array}{l}\text { Thickness } \\
{[\mathrm{cm}]}\end{array}$ & $\begin{array}{l}\text { Wavelength } \\
{[\lambda]}\end{array}$ \\
\hline Skin & 0.15 & 0.012 \\
\hline Subcutaneous fat & 2.5 & 0.0033 \\
\hline Muscle & 1.2 & 0.010 \\
\hline Visceral fat & 2.0 & 0.0033 \\
\hline SI wall & 0.1 & 0.010 \\
\hline SI content & 2.0 & 0.010 \\
\hline
\end{tabular}

\section{Propagation through fat layers with 2D power flows}

In this section, we evaluate the propagation through the fat layer by studying the $2 \mathrm{D}$ power flow diagrams and Poynting vector values in different areas of the abdomen area. This study is continuation for power distribution studies presented in [10], however, now we focus especially on the propagation through the fat layer.

First, we study the 2D power flow representations for the horizontal and vertical cross sections as presented in Fig. 3a-b. The power flow is normalized for $-60 \mathrm{~dB}-$ $0 \mathrm{~dB}$ range for the clarity. Similar power flow figures are presented in the in-body power distribution results in [10] but they are repeated here for the fat propagation channel analysis. The power flow arrow representation describes well how the signal propagates in the vicinity and inside the human body. Signal propagates along the body surface, as creeping waves [25], but on the way, the part of the signal also passes the skin surface and propagates inside the tissues. One can easily notice the propagation through the fat layer both in the horizontal and vertical cross sections. In the horizontal cross section, the back area can easily be reached within the maximum attenuation $-60 \mathrm{~dB}$ as the signal propagates through the subcutaneous fat layer. Instead, attenuation is strong in the intestine area and thus no power arrays are visible behind the intestine area. From the vertical cross section in Fig. 3b, one can easily note how the propagation travels vertically through the fat layer over a wide area. Within the plotted $\mathrm{dB}$ range from $-60 \mathrm{~dB}$ to $0 \mathrm{~dB}$, the area from the upper parts of the leg until the stomach can be covered.

Next, we will investigate the power values in different parts of the subcutaneous fat as well as in the intestine area. Table III summarizes the relative power values at $f=4 \mathrm{GHz}$. The values are normalized so that the power is $0 \mathrm{~dB}$ just before the skin layer below the Tx-antenna. This way we can easily observe the propagation loss within the tissues. Points A- $\mathrm{H}$ correspond the power values in the fat layer on the path from the navel to the back. In the point $\mathrm{A}$, the power is $-4 \mathrm{~dB}$ and at $\mathrm{H}$ is $-74 \mathrm{~dB}$. As the power flow arrows describe, the signal in the fat layer consist of the signal passing through the skin (from creeping waves) as well as the signal propagated through fat layer from the antenna. The power decreases gradually as the distance from the Tx antenna increases. Surprisingly, the power is at the points F and G $-48.5 \mathrm{~dB}$ and $-48.0 \mathrm{~dB}$, respectively. The power is slightly higher at $\mathrm{G}$, since there is 
more power summed from the larger area of propagation through the fat layer compared to the point $\mathrm{F}$, whose power consists mostly the power arriving through the skin.

Next, we compare the power values inside different parts of the intestine and visceral fat area. It is interesting to note how the power is remarkable higher in the points on the visceral fat area compared to the points on the intestine area although the distance from the transmitter antenna is the same. For instance, in the furthest point of the small intestine $\mathrm{M}$ the power is $-68 \mathrm{~dB}$, whereas in the visceral fat point $\mathrm{N}$, which is next to $\mathrm{M}$, the power is $-54 \mathrm{~dB}$. It is also interesting to note that the power in $\mathrm{G}$ is approximately $20 \mathrm{~dB}$ higher than in $\mathrm{M}$, although $\mathrm{M}$ is clearly closer to the Tx antenna than point G. Similarly, as we compare visceral fat point value $\mathrm{P}$ and small intestine point $\mathrm{K}$, we can note that the power is $5 \mathrm{~dB}$ higher at $\mathrm{P}$ than in $\mathrm{K}$. At the visceral fat point $\mathrm{L}$ and SI point $\mathrm{K}$, the difference is only $2 \mathrm{~dB}$.

The impact of the fat to the propagation path in the signal strength can easily be noticed when evaluating power values in the left colon points: R, S, T, and U. At the point $\mathrm{R}$, where part of the signal has a direct path from the on-body antenna through the fat layer, the power is $-16 \mathrm{~dB}$. Whereas at point $\mathrm{S}$, the power level is $-32 \mathrm{~dB}$. The loss in the colon tissue is high and, thus, the power difference between the points $\mathrm{R}$ and $\mathrm{S}$ are remarkable. At the point $\mathrm{T}$, the power is again higher, $-22 \mathrm{~dB}$, since the signal can reach the colon from the left side of the abdominal muscle directly through the fat layer. At the points $\mathrm{U}$ and $\mathrm{V}$, the power is again significantly lower: $-36 \mathrm{~dB}$ and $-41 \mathrm{~dB}$, respectively.

These power studies verify that the signal propagates with minor losses in the fat tissue. Additionally, the averaged power is higher in the small intestine points, which are in the vicinity of the subcutaneous or visceral fat. 

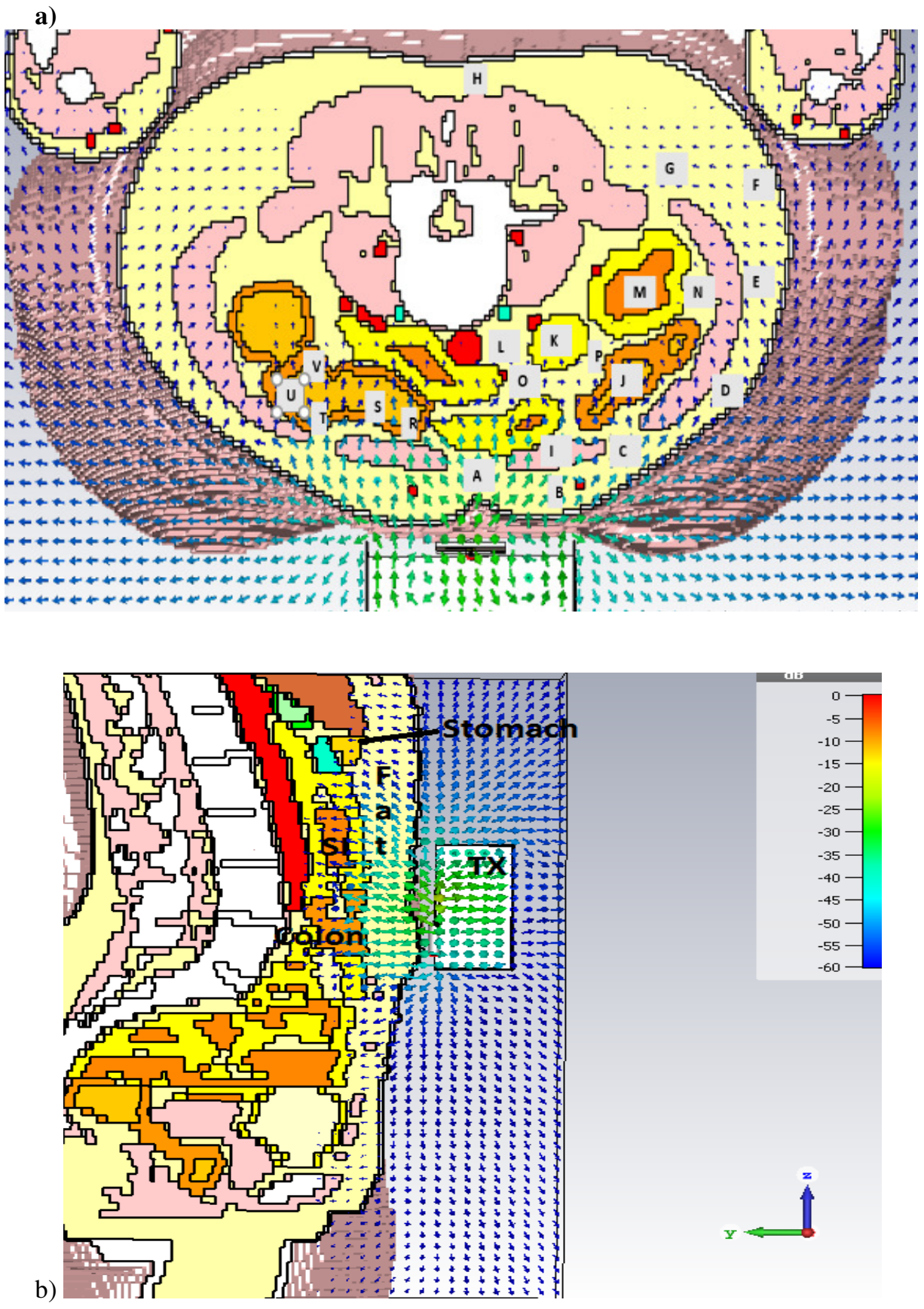
Fig. 3 a) Power flow diagram of the on the cross-cut A with the antenna location option $1, \mathrm{~b})$ power flow diagram with the vertical cross section

Table III Power at different points in the abdominal area.

\begin{tabular}{|l|c|c|c|c|c|c|c|c|}
\hline $\begin{array}{l}\text { Subcutaneous } \\
\text { fat points }\end{array}$ & A & B & C & D & E & F & G & H \\
\hline Power [dB] & -4 & -5 & -18 & -33 & -43 & -48.5 & -48.1 & -74 \\
\hline $\begin{array}{l}\text { GI/Visceral } \\
\text { fat points }\end{array}$ & I & J & K & L & M & N & O & P \\
\hline Power [dB] & -10 & -40 & -51 & -49 & -68 & -54 & -39 & -46 \\
\hline Colon points & R & S & T & U & V & & & \\
\hline Power [dB] & -16 & -32 & -22 & -36 & -41 & & & \\
\hline
\end{tabular}

\section{Propagation through subcutaneous fat with the channel simulations and measurement data}

In this section, we evaluate the propagation through the fat layer by comparing the propagation path calculations to the simulated and measured data. The part of the data has been published in [7], in which we present the channel characteristics on the abdomen area. Some of the impulse responses are repeated here to ease the analysis for the fat as a propagation medium but also to ease the comparison between the channel responses and propagation path calculations.

\subsection{Simulation results}

First, we evaluate the channel characteristics using Gustav-voxel model and verify it with the corresponding propagation path calculations. Propagation paths are calculated using the dimensions obtained from the Gustav's cross section, presented in Fig. 4. The thicknesses of the subcutaneous fat layers below the Tx antenna (on the navel) is $d \mathrm{f}=2.5 \mathrm{~cm}$, and below the Rx antenna (on the flank) is $d \mathrm{~s}=2 \mathrm{~cm}$. Based on the power flow figures presented in the previous section, the signal is assumed to travel through the subcutaneous fat layer, both outer part of the subcutaneous fat (Path1) as well as inner part of the subcutaneous fat (Path2). Propagation distance of Path1 is estimated to be $\mathrm{d} 1=25.5 \mathrm{~cm}$. Propagation distance of Path2 $\left(d_{2}\right)$ is estimated to be $d 2=d 1+d \mathrm{~s}+d \mathrm{f}$. Obviously, there is a propagation between these limits, in the middle part of the fat layer. Besides there is a path, in which the signal travels cross the subcutaneous fat layer towards intestine area until reaching the visceral fat layer, and then travels via visceral fat until reaching the area where the abdominal muscle layer is thinner, and returns back to the subcutaneous fat layer continuing towards the Rx antenna on the flank. Path 3 is the propagation path, which travel around the abdominal muscle on the right side and then merges to the Path 2 in the point A. The length of this round trip in the visceral fat layer $d$ sur is estimated to be $8 \mathrm{~cm}$ and hence, the total length of the Path3 is $d 3=d 2^{\prime}+d$ sur, where $d 2^{\prime}$ is the distance from the point 
A until the Rx antenna via Path 2. Additionally, the signal can travel similarly around the left abdominal muscle resulting in the $d 4=d 2+d$ sul, which is denoted as Path 4 .

Next, the propagation times are calculated for the explained propagation paths. The propagation times are summarized in Table IV. For the value presented in the Table II, we have added the propagation time which $t$ skin $=2 * 0.06 \mathrm{~ns}$, which takes when the signal passes the skin layer until the fat layer. Now, when we compare the obtained propagation times with the IRs presented in Fig. 5, we can see a clear correspondence between the location of the strongest peaks of the IR and the calculated propagation times. In this antenna location option, there is no clear line-of-sight (LOS) component since the antennas are towards the different directions. Thus, the IR consists of the peaks arriving through the creeping waves [25] as well as via the propagation within the tissues. In this study, we focus on the propagation through fat layer. The strongest peak of the IR arrives at time instant of $2 \mathrm{~ns}$, which corresponds to the propagation path through the outer subcutaneous fat Path1. The width of the first peak is $1.1 \mathrm{~ns}$, starting from $1.5 \mathrm{~ns}$ until $2.6 \mathrm{~ns}$. Propagation time for the Path2 is calculated to be $2.4 \mathrm{~ns}$, which is inside the first peak's range. The second strongest peak arrives at $2.4 \mathrm{~ns}$, which corresponds to the Path3. Propagation time for Path 4 is calculated to be $3.8 \mathrm{~ns}$, which also can be found from the IR. The rest of the IR peaks belong to the propagation paths travelling through other tissues as well as propagation via body surface, but those are out of scope in this paper.

The obtained propagation paths match well with the peaks appearing in the simulated IR. Some of the peaks are wider than the others. This is because there are several indistinguishable paths in the fat layer as well. Signal may bounce back and forth from the muscle and SI layers making the propagation distance slightly longer. Next, we will verify the simulated results with the measurement data.

Table IV Propagation times with Gustav-voxel

\begin{tabular}{|l|c|c|c|c|}
\hline Paths through the fat & Path1 & Path2 & Path3 & Path4 \\
\hline $\begin{array}{l}\text { Propagation distance } \\
\text { in fat [cm] }\end{array}$ & 25.5 & 31 & 34 & 50 \\
\hline $\begin{array}{l}\text { Propagation time } \\
\text { [ns] }\end{array}$ & 2 & 2.4 & 2.6 & 3.8 \\
\hline
\end{tabular}




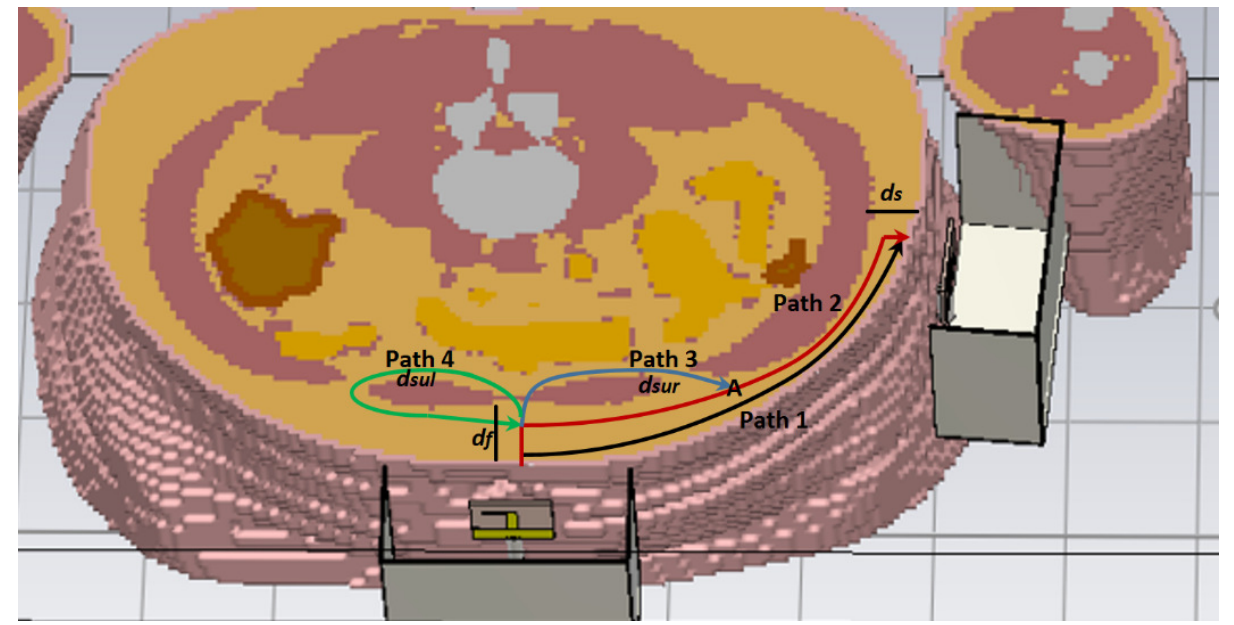

Fig. 4 Dimensions on Gustav-voxel's cross section and propagation path 1-4.

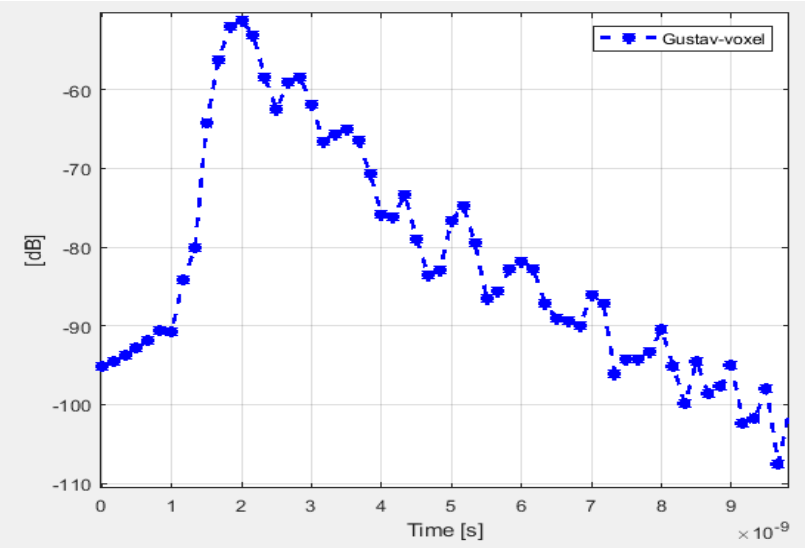

Fig. 5 Impulse response obtained with the Gustav-voxel.

\subsection{Measurement results}

In the measurements, the antenna separation distance for the female volunteer F1 was $24.5 \mathrm{~cm}$ and for the male volunteer M3 is $27.5 \mathrm{~cm}$. The differences in the distances are due the different sizes of the waists of the volunteers. The distances $d$ s and $d \mathrm{f}$ are estimated in this case since there was no tools to measure exact thicknesses of the subcutaneous fat layers of the volunteers. The corresponding dimensions of the voxel models were used to estimate the dimensions. For the volunteer F1, $d$ s and $d$ f were estimated to be $2.5 \mathrm{~cm}$ and for the volunteer $\mathrm{M} 3,6 \mathrm{~cm}$. Additionally, $d$ sur and $d$ sul are estimated to same as for the Gustav model, $7 \mathrm{~cm}$ and $8 \mathrm{~cm}$, respectively.

Propagation times are summarized in Table V. Next, we compare the calculated propagation times presented in Table III with the IRs obtained with different volunteers which are presented in Fig.3. As in the case of the simulation results, there is no 
clear line-of-sight (LOS) component between the antennas. By free-space propagation calculations, the LOS signal should arrive at the time instants $0.8 \mathrm{~ns}$ and $0.9 \mathrm{~ns}$ for the antenna separation distances $24.5 \mathrm{~cm}$ and $27.5 \mathrm{~cm}$, respectively. The impulse responses consist of the peaks due to creeping waves [25] as well as propagation within the tissues.

The Path1(outer part of the subcutaneous fat) is calculated to arrive for the volunteers F1 and M3 at the time instants $1.8 \mathrm{~ns}$ and $2.2 \mathrm{~ns}$, as we take account also the time when the signals pass the skin layer as well (at $0.06 \mathrm{~ns}$ ). As one can note from the Fig. 6, there is a clear peak at that time instant for the volunteers. Based on the propagation path calculations, Path2 would arrive for the volunteers M3 and F1 at $3.0 \mathrm{~ns}$ and $2.4 \mathrm{~ns}$, respectively. These peaks can also be clearly found from the both IRs as well. Path3 and Path4 arrive for the volunteer F1 at $2.6 \mathrm{~ns}$ and $3.5 \mathrm{~ns}$ and for the M3 the both paths 3 and 4 at $3.6 \mathrm{~ns}$. Also, in this case the match with the measured IRs is good. Additionally, in the measured IRs, one can note several peaks around these time instants.

The reason for this is that the signal travels through the whole fat layer, not just inner and outer fat layers, which can be seen as several peaks one after another. Volunteer M3 has more peaks at the time range 3-4.5 ns and besides the strength of the peaks are clearly higher than those of the volunteer F1. The larger number of the peaks are assumed to be due to the thicker subcutaneous and visceral fat layers the volunteer M3 has. Furthermore, there is clear difference of the level of the IR peaks: M3's peaks are remarkably higher than those of the F1. The reason for this may be just the tilting of the antennas: Even the small tilts towards different direction can significantly decrease the received signal strength between the Tx-Rx link, especially in this kind of antenna configuration. This appear due to the antenna directivity [24].

There is surprisingly good correspondence between the measurement results and propagation path calculations. Naturally, the dimension relating to the visceral fat layer are just estimated values and thus there is some uncertainties in the data. The knowledge about the fat as a propagation channel is useful when designing the monitoring antennas and especially the antenna locations for on-in body communication links. The signal may travel from the intestine area through the visceral and subcutaneous fat with reasonable losses if the antennas are located in the area where there is less muscle layer between the visceral and subcutaneous fat layers. 


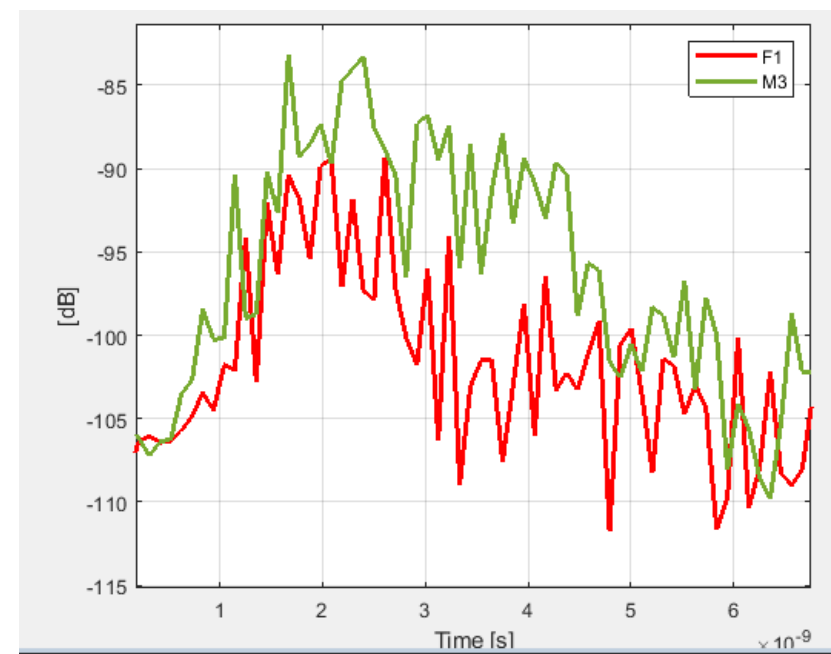

Fig. 6 Impulse response obtained with the volunteer F1 and M3.

Table V Propagation times in the fat layer for the volunteers F1 and M3

\begin{tabular}{|c|c|c|c|c|}
\hline Paths through the fat & Path1 & Path2 & Path3 & Path4 \\
\hline Propagation distance & F1:25.5 & F1:31.5 & F1:34.5 & F1: 45.5 \\
in fat [cm] & M3:27.5 & M3:44.5 & M3: 47.5 & M3: 47.5 \\
\hline \multirow{2}{*}{ Propagation time [ns] } & F1: 1.8 & F1: 2.4 & F1: 2.6 & F1: 3.5 \\
& M3: 2.2 & M3: 3 & M3: 3.6 & M3: 3.6 \\
\hline
\end{tabular}

\section{Conclusions}

This paper presents a study of the fat in the abdomen area as a propagation medium in WBAN applications. Propagation via subcutaneous and visceral fat is considered separately. The study was conducted via simulations with anatomical voxel models evaluating the channel characteristics between two on-body antennas designed for inbody communications as well as via corresponding on-body measurements. Simulations and measurements were done for both female and male bodies with the on-body antennas designed for in-body communications. Propagation paths were calculated and compared with the simulated and measured impulse responses. Furthermore, we analyzed simulated $2 \mathrm{D}$ power flow figures, which illustrate the propagation inside the different tissues.

It was shown that the signal propagates through fat layer with minor loss compared to the other tissues. The signal propagates through the fat tissue from the abdomen area to the backside of person with $60 \mathrm{~dB}$ power loss. Additionally, the timing of the calculated propagation paths through the fat layer match well with the peaks of the simulated and measured impulse responses.

The knowledge about the fat as a propagation medium is useful when designing the wireless and wired medical and health monitoring devices. Location of the anten- 
nas/sensor nodes for the monitoring devices can be establish so that the propagation through the fat layer can be maximized. Appropriate antenna locations can also facilitate that the signal may reach deeper inside the abdominal tissues via subcutaneous and visceral fat, which is useful for the implant communication systems. As a future work, we plan to continue the study by using the simulation models having different thickness of the subcutaneous and visceral fat. Furthermore, more measurements will be conducted with different antenna locations and with several volunteers having different body balance.

\section{Acknowledgements}

This research has been financially supported by the project WBAN Communications in the Congested Environments and in part by Academy of Finland 6Genesis Flagship (grant 318927). Ilkka Virtanen, Timo Mäkinen, and Jari Sillanpää from University of Oulu deserve acknowledgement for their help to enable the exhaustive simulations. Dr. Sami Myllymäki is acknowledged for preparing the prototypes.

\section{References}

1. A. Teshome, B. Kibret, D. T. H. Lai, "A Review of Implant Communication Technology in WBAN, Progresses and Challenges," IEEE Reviews in Biomedical Engineering, 2018.

2. E. Schires, P. Georgiou, T. S. Lande, "Vital Sign Monitoring Through Back Using an UWB Impulse Radar with Body Coupled Antennas," IEEE Transaction on Biomedical Circuits and Systems, 2018.

3. Y. Wei, A. Zahid, H. Heidari, M. Imran, Q. H. Abbasi, A Compact Non- Invasive Wearable Vital Signal Monitoring System," IEEE Asia Pasific Conference on Postgraduate Research in Microelectronics and Electronics, 2018.

4. P. Leelatien, K. Ito, K. Saito, M. Sharma, A. Alomainy, "Channel Characteristics and Wireless Telemetry Performance of Transplanmted Organ Monitoring System Using Ultrawideband Communication," IEEE Journal of Electromagnetics, RF and Microwaves in Medicine and Biology, 2018.

5. M. Särestöniemi, C. Pomalaza-Raez, T. Kumpuniemi, M. Hämäläinen, J. Iinatti, "Measurement Data Based Study on the Intra-Body Propagation in the Presence of the Sternotomy Wires and Aortic Valve Implant," Transaction on Antennas and Propagation, 2019.

6. M. Särestöniemi, C. Pomalaza Raez., Z. Bi, T. Kumpuniemi, C. Kissi, M. Sonkki, M. Hämäläinen, J. Iinatti, "Comprenhensive Study on the Impact of the Sternotomy Wires on the UWB WBAN Channel Characteristics," IEEE Access, 2019.

7. M. Särestöniemi; C. Kissi;C. Pomalaza-Raez; T. Kumpuniemi ;M. Sonkki; S. Myllymäki ; M. Hämäläinen ; and J. Iinatti, "Measurement and simulation based study on the UWB channel characteristics on the abdomen area", ISMICT 2019.

8. M. Särestöniemi; C. Kissi; C. Pomalaza-Raez ; M. Hämäläinen; and J. Iinatti, "Impact of the antenna-body distance on the UWB on-body channel characteristics", ISMICT 2019.

9. M. Särestöniemi; C. Kissi; C. Pomalaza Raez;. M. Hämäläinen and J. Iinatti, "Propagation and UWB channel characteristics on human abdomen area," EUCAP 2019. 
10. M. Särestöniemi, C. Pomalaza-Raez, M. Berg, C. Kissi, M. Hämäläinen. J. Iinatti, ’InBody Power Distribution for Abdominal Monitoring and Implant Communications Systems," accepted to be published in ISWCS, September 2019.

11. A. F. Demir, Q. H. Abbasi, Z. E. Ankarali, A Alomainy, K.Qaraqe, E. Serpedin, H. Arsalan, "Anatomical Region-Specific In Vivo Wireless Communication Channel Characterization," IEEE Journal on Biomedical and Health Informatics, 2017.

12. R. Chávez-Santiago et al., "Experimental path loss models for in-body communications within 2.36-2.5 GHz", IEEE Journal on Biomedical Health Informatics, 2015.

13. J. Li, Z. Nie, Y. Liu, L. Wang, Y. Hao, "Characterization of In-Body Radio Channels for Wireless Implants," IEEE Sensors Journal, 2017.

14. A. Khaleghi, I. Balansingham, R. Chavez-Santiago, "Computational Study of Ultra Wideband Wave Propagation into the Human Chest," IET Microwaves, Antennas \& Propagation, 2009,

15. P. Turalchuk, I. Munina, V. Pleskachev, V. Kirillov, O. Vendik, I. Vendik, "In-Body and On-Body Wave Propagation: Modeling and Measurements," International Workshop on Antenna Technology: Small Antennas, Innovative Structures, and Applications(iWAT), 2017.

16. Y. El-Saboni, G. A. Conway, S. L. Cotton, W. G. Scanlon, "Radiowave Propagation Characteristics in the Intra_Body Channel at $2.38 \mathrm{GHz}$ ", IEEE International Conference on Wearable and Implantable Body Sensor Networks (BSN), 2017.

17. A. Alomainy, Y. Hao, Y. Yuan, Y. Liu, "Modelling and Characterization of Radio Propagation from Wireless Implants at Different Frequencies," European Coneference on Wireless Technology, 2009.

18. https://www.itis.ethz.ch/virtual-population/tissue-properties/database

19. N. B. Asan, et al. Intra-body Microwave Communication Through Adipose Tissue, healthcare Technology Letter, 2017.

20. N. B. Asan et al. Characterization of Fat Channel for intra-Body Communication at RBand Frequencies, MDPI Sensors, 2018.

21. N. B. Asan, J. Velander, S. Redzwan, R. Augustine, E. Hassan, D. Noreland, T. Voigt, T. J. Blokhuis, "Reliability of the Fat Tissue Channel for Intra-Body Microwave Communication,".

22. CST Microwave Studio, [Online]. Available: http://www.cst.com

23. IEEE Standard for Local and metropolitan area networks _Part 15.6: Wireless Body Area Networks, pp. IEEE Std 802.15.6-2012, pp. 1 - 271, 2012.

24. C. Kissi; M. Särestöniemi; C. P-. Raez; M. Sonkki; and M. N. Srifi, "Low-UWB directive antenna for Wireless Capsule Endoscopy localization”, BodyNets2018.

25. T. Kumpuniemi, M. Hämäläinen, J-P. Mäkelä, J. Iinatti, "Path Loss Modeling for UWB Creeping Waves Around Human Body, ’ISMICT2017, Portugal. 